\title{
Influência da fisioterapia respiratória sobre a função cardiopulmonar em recém-nascidos de muito baixo peso
}

\author{
Effects of respiratory physiotherapy on the cardiopulmonary function of very low birth weight newborn \\ infants
}

Carla Marques Nicolau ${ }^{1}$, Mário Cícero Falcão

\section{RESUMO}

Objetivo: Avaliar as repercussões da fisioterapia respiratória sobre a função cardiopulmonar em recém-nascidos pré-termo (RNPT) submetidos à ventilação mecânica.

Métodos: Estudo prospectivo de RNPT com peso de nascimento menor que $1500 \mathrm{~g}$, sendo verificados os valores de frequência cardíaca (FC), saturação de oxigênio $\left(\mathrm{SatO}_{2}\right)$, frequência respiratória (FR) e pressão arterial sistêmica (PA) antes e após a fisioterapia respiratória e a aspiração endotraqueal. Os RNPT incluídos foram avaliados em sessões sequenciais entre o $3^{\circ}-7^{\circ}$ dias de vida por dois fisioterapeutas da unidade neonatal. Os valores de $\mathrm{FC}, \mathrm{SatO}_{2}$ e PA foram coletados por monitorização eletrônica e a FR, por cronômetro. $\mathrm{Na}$ análise estatística, foi utilizado o teste de ANOVA para medidas repetidas, sendo significante $p<0,05$.

Resultados: Foram estudados 42 recém-nascidos, $57 \%$ do sexo feminino, com peso de nascimento médio de $1024 \mathrm{~g}$ e idade gestacional média de 29,5 semanas. Somente 3 RNPT apresentaram Apgar de $5^{\circ}$ minuto menor que 5 e a Doença das Membranas Hialinas foi o principal diagnóstico respiratório (88\%). Foram realizadas 252 sessões de fisioterapia. A FC, FR, $\mathrm{SatO}_{2}$ e PA atingiram valores considerados fisiológicos após os procedimentos fisioterapêuticos e a aspiração endotraqueal.

Conclusões: Os procedimentos de fisioterapia respiratória e de aspiração endotraqueal não apresentaram influências significativas na função cardiopulmonar, sugerindo que, quando bem indicados e realizados, não comprometem a estabilidade clínica de RNPT.

Palavras-chave: recém-nascido; prematuro; fisioterapia; monitorização fisiológica.

\section{ABSTRACT}

Objective: To assess the effects of respiratory physiotherapy on cardiopulmonary function of ventilated preterm newborns infants (PTNB).

Methods: This prospective study included PTNB with birth weight lower than $1500 \mathrm{~g}$. Before and after respiratory physiotherapy and endotracheal suction, the following parameters were evaluated: heart rate (HR), oxygen saturation $\left(\mathrm{O}_{2} \mathrm{Sat}\right)$, respiratory rate $(\mathrm{RR})$ and blood pressure (BP). The newborns were evaluated in consecutive sessions between the $3^{\text {rd }}-7^{\text {th }}$ day of life by two therapists in the neonatal unit. $\mathrm{HR}, \mathrm{O}_{2}$ Sat and BP data were collected by electronic monitoring and RR by a stopwatch. Results were analyzed by repeated measures ANOVA, being significant $p<0.05$.

Results: We evaluated 42 PTNB, 57\% females, with a mean birth weight of $1024 \mathrm{~g}$ and a mean gestational age of 29.5 weeks. Only three PTNB had a $5^{\text {th }}$ minute Apgar lower than 5. Respiratory Distress Syndrome was the most prevalent disease (88\%). In total, 252 physiotherapy sessions were applied to these 42 infants. $\mathrm{HR}, \mathrm{RR}, \mathrm{O}_{2}$ Sat and BP remained within the physiological limits after procedures, including endotracheal suction.

Conclusions: Procedures for physiotherapy and endotracheal suction showed no significant influences on cardiopulmonary function, suggesting that, when properly carried out, they do not compromise the clinical stability of PNTB.

Key-words: infant, newborn; infant, premature; physical therapy; monitoring, physiologic.

Endereço para correspondência:

Carla Marques Nicolau

Rua Gabriel de Brito, 332 - Cerqueira Cesar

CEP 05411-010 - São Paulo/SP

Email: carla.nicolau@icr.usp.br ou carlanicolau@ig.com.br

Conflitos de interesse: nada a declarar

Recebido em: 14/7/09

Aprovado em: 16/9/09 


\section{Introdução}

O contínuo desenvolvimento tecnológico e científico na área da assistência perinatal e neonatal nas últimas décadas tem sido fator determinante para o decréscimo da mortalidade e da morbidade neonatal. $\mathrm{O}$ aumento da sobrevida de recém-nascidos (RN) com peso de nascimento e idade gestacional cada vez mais baixos fez com que aumentasse o período de hospitalização destes pacientes, devido principalmente à imaturidade pulmonar ${ }^{(1-3)}$.

O desenvolvimento contínuo da fisioterapia nas unidades neonatais faz com que as técnicas e os recursos fossem aprimorados de forma específica para essa população-alvo, contribuindo para reduzir a morbidade neonatal ${ }^{(2,3)}$, o tempo de hospitalização ${ }^{(4,5)}$ e os custos hospitalares ${ }^{(6)}$. Contudo, as evidências para avaliar o risco-benefício da fisioterapia respiratória em recém-nascidos pré-termo (RNPT) são restritas, já que os estudos são escassos, possuem amostras pequenas e desenhos experimentais díspares ${ }^{(2,7-9)}$.

Simultaneamente, constataram-se alguns efeitos adversos da fisioterapia, especialmente nos prematuros, o que ensejou uma escolha mais criteriosa dos procedimentos fisioterapêuticos, que passaram a ter avaliação e execução individualizadas, de acordo com o paciente. Há uma tendência, atualmente, de se evitarem manipulações frequentes e/ou intensas no RNPT pelo risco de efeitos adversos ${ }^{(3,8-10)}$.

Nesse contexto, a proposta do presente estudo foi avaliar a influência da fisioterapia respiratória e da aspiração endotraqueal sobre a função cardiopulmonar (frequência cardíaca, frequência respiratória, saturação de oxigênio e pressão arterial sistêmica) em RNPT de muito baixo peso submetidos à ventilação mecânica na primeira semana de vida.

\section{Métodos}

O estudo clínico foi conduzido de forma prospectiva, incluindo RNPT (idade gestacional inferior a 37 semanas), após a assinatura do termo de consentimento pelos pais ou pelo responsável. Este estudo foi aprovado pela Comissão de Ética para Análise de Projetos de Pesquisa.

Foram incluídos RNPT de ambos os sexos, com peso de nascimento menor que $1500 \mathrm{~g}$, idade pós-natal entre três e sete dias, em ventilação mecânica. Foram excluídos os RN portadores de malformações congênitas graves, com síndromes genéticas, além das situações clínicas em que a fisioterapia respiratória estava contraindicada (pneumotórax não drenado, hipertensão pulmonar diagnosticada em exame de ecocardiograma, hemorragia intracraniana e coagulopatias).

O estudo foi desenvolvido na unidade de terapia intensiva neonatal do Berçário Anexo à Maternidade do Instituto da Criança do Hospital das Clínicas da Faculdade de Medicina da Universidade de São Paulo, no período de fevereiro de 2003 a setembro de 2004, preenchendo-se um formulário para cada $\mathrm{RN}$ incluído no estudo por consulta de prontuários, coletando-se as seguintes informações: data de nascimento, tipo de parto, sexo, idade gestacional (em semanas), peso de nascimento (em gramas), Boletim de Apgar de $1^{\circ}, 5^{\circ} \mathrm{e}$ $10^{\circ}$ minutos de vida, o escore CRIB (Clinical Risk Index for Babies) e os diagnósticos clínicos, com ênfase nas afecções respiratórias.

A determinação dos valores da frequência cardíaca e da saturação de oxigênio foi realizada por meio do sistema de monitorização de sinais vitais DX 2010-LCD - Dixtal Biomédica ${ }^{\circledR}$; a frequência respiratória foi determinada por cronômetro. A pressão arterial sistêmica foi mensurada por medida de pressão arterial não-invasiva - método oscilométrico com manguito próprio - Sistema de monitorização de sinais vitais DX 2010-LCD - Dixtal Biomédica ${ }^{\circledR}$. O manguito utilizado foi de tamanho apropriado para a circunferência e o comprimento do membro do RN. Dos métodos não-invasivos de medida da pressão arterial, o oscilométrico é o mais utilizado no período neonatal devido à ausência de complicações, fidedignidade e facilidade de manipulação ${ }^{(11)}$.

Os procedimentos fisioterapêuticos utilizados compreenderam um protocolo padronizado de atendimento: adoção do posicionamento nos decúbitos lateral direito e esquerdo associado com a vibração manual, apoio diafragmático e das últimas costelas e o procedimento de aspiração endotraqueal.

Os valores das variáveis estudadas (frequência cardíaca e respiratória, saturação de oxigênio e pressão arterial sistêmica) foram registrados antes do início de cada sessão de fisioterapia (T0). Os RNPT receberam os procedimentos fisioterapêuticos por um período máximo de dez minutos. As mesmas variáveis foram novamente mensuradas imediatamente após (T1) e cinco minutos após os procedimentos de fisioterapia respiratória (T2). A aspiração endotraqueal e/ou de vias aéreas superiores foi realizada sempre cinco minutos após os procedimentos fisioterapêuticos, sendo verificados os valores das variáveis estudadas nos momentos imediatamente após (T3) e cinco minutos após o procedimento de aspiração (T4). O procedimento de aspiração foi realizado em todos os $\mathrm{RN}$ do estudo de forma asséptica, 
com o sistema aberto de aspiração, introduzindo-se a sonda de aspiração por duas vezes em cada RN. Segundo a normatização da unidade, durante o procedimento de aspiração aumentou-se a fração inspirada de oxigênio em $10 \%$.

O registro dos valores das variáveis estudadas foi realizado pelas enfermeiras da unidade neonatal nos momentos previamente estabelecidos; a realização das sessões de fisioterapia foi executada por dois fisioterapeutas da unidade neonatal, seguindo-se a padronização do atendimento de fisioterapia adotada para a realização do estudo, de acordo com a rotina da escala desses profissionais.

As variáveis foram controladas nas primeiras seis sessões de fisioterapia realizadas entre o terceiro e o sétimo dia de vida, compreendendo o período mais crítico da evolução dos RNPT. Todos os $\mathrm{RN}$ receberam um mínimo de seis sessões de fisioterapia entre o terceiro e o sétimo dia de vida, sendo esta realizada sempre por um fisioterapeuta.

O tamanho da amostra foi calculado para detectar uma diferença de aproximadamente $10 \%$ nas médias das frequências cardíaca e respiratória, da saturação de oxigênio e da pressão arterial sistêmica (sistólica, diastólica e média) ${ }^{(11)}$. Para um alfa de $5 \%$ e um poder de teste de $80 \%$, o número de pacientes necessários calculado foi de $40 \mathrm{RN}$.

Os dados foram submetidos à análise descritiva, avaliando-se a distribuição de cada variável estudada. Os dados nominais foram descritos em termos de porcentagens e proporções. A comparação de cada variável estudada de acordo com o momento avaliado e a evolução entre as sessões foi feita através do teste de análise de variância para medidas repetidas (ANOVA-MR oneway). Os resultados foram considerados estatisticamente significante se $p<0,05$. Os dados foram processados no software Sigma Stat.

\section{Resultados}

Foram incluídos no estudo 48 RNPT. Destes, seis foram excluídos a posteriori após a confirmação diagnóstica de hipertensão pulmonar. As características da população estudada estão ilustradas pela Tabela 1 .

Todos os RN foram ventilados mecanicamente em respiradores de fluxo contínuo, limitados à pressão e ciclados a tempo, no modo ventilação mandatória intermitente (IMV). Dos 42 RN estudados, 37 (88\%) receberam uma dose de surfactante exógeno nas primeiras seis horas de vida. Nenhum dos RN incluídos no estudo fazia uso de
Tabela 1 - Características da população estudada $(n=42)$

\begin{tabular}{|c|c|}
\hline Variável & $\begin{array}{c}\text { média } \pm \text { DP } \\
\text { ou n }(\%)\end{array}$ \\
\hline Idade gestacional (semanas) & $29,5 \pm 2,1$ \\
\hline Peso de nascimento $(\mathrm{g})$ & $1024 \pm 281$ \\
\hline \multicolumn{2}{|l|}{ Sexo } \\
\hline Feminino & $24(57 \%)$ \\
\hline Masculino & $18(43 \%)$ \\
\hline \multicolumn{2}{|l|}{ Adequação nutricional } \\
\hline Adequado para a idade gestacional & $33(78 \%)$ \\
\hline Pequeno para a idade gestacional & $9(22 \%)$ \\
\hline Apgar $5^{\circ} \min <5$ & $3(7 \%)$ \\
\hline \multicolumn{2}{|l|}{ Principal afecção respiratória } \\
\hline Síndrome do desconforto respiratório & $37(88 \%)$ \\
\hline Síndrome do pulmão úmido & $5(12 \%)$ \\
\hline \multicolumn{2}{|l|}{ Clinical Risk Index for Babies } \\
\hline $0-5$ & $25(59 \%)$ \\
\hline $6-10$ & $15(36 \%)$ \\
\hline$>10$ & $2(5 \%)$ \\
\hline
\end{tabular}

sedativos e/ou analgésicos, drogas vasoativas e/ou anticonvulsivantes.

Nesses $42 \mathrm{RN}$, foram realizadas 288 sessões de fisioterapia. Entretanto, para a tabulação dos dados, foram considerados os seis primeiros atendimentos de cada RNPT, totalizando 252 sessões de fisioterapia, incluindo a aspiração de vias aéreas. Essas sessões foram realizadas de duas a três vezes por dia, conforme a rotina do Serviço, justificando-se a escolha da primeira semana de vida como critério de inclusão.

As variações das médias dos parâmetros estudados (frequência cardíaca e respiratória, saturação de oxigênio e pressão arterial sistêmica), de acordo com o momento analisado, estão referidas nas Figuras 1 a 4 . As análises em relação à evolução das variáveis estudadas de acordo com as sessões de fisioterapia mostraram variação semelhante das médias das frequências cardíaca e respiratória, da saturação de oxigênio e da pressão arterial sistêmica (sistólica, diastólica e média) entre todas as seis sessões realizadas, não sendo observadas diferenças estatisticamente significativas.

\section{Discussão}

A fisioterapia respiratória neonatal vem ganhando especial atenção por parte dos pesquisadores, em virtude do crescente aumento da sua indicação e aplicação nos últimos anos. No entanto, há muita controvérsia sobre o real papel 


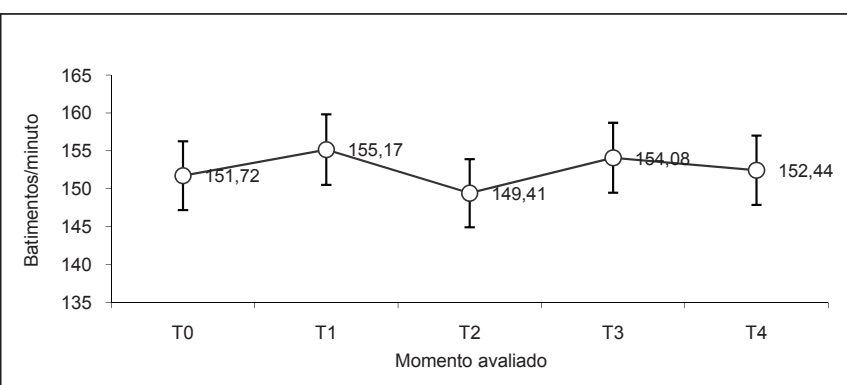

ANOVA MR: T1xT2 p<0,001; T2xT3 $p<0,001 ; \mathrm{T} 2 \mathrm{xT} 4 p<0,001$.

Figura 1 - Média da frequência cardíaca dos recém-nascidos estudados, de acordo com o momento avaliado.

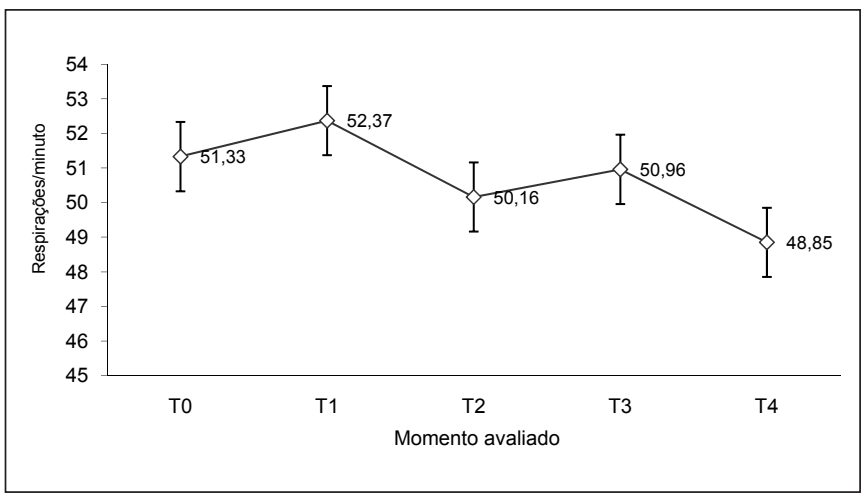

Figura 2 - Média da frequência respiratória dos recém-nascidos estudados, de acordo com o momento avaliado.

desta atividade de maneira sistemática nas unidades neonatais. A literatura recomenda uma ação fisioterapêutica mais cautelosa e individualizada, de acordo com a necessidade de cada $\mathrm{RN}^{(1)}$.

No presente estudo, foram incluídos predominantemente $\mathrm{RN}$ prematuros extremos (idade gestacional inferior a 30 semanas), com adequação nutricional e valores do Clinical Risk Index for Babies (CRIB) entre 0 e 5 . A introdução de tecnologias sofisticadas no tratamento da insuficiência respiratória do $\mathrm{RN}$, como o surfactante exógeno, o óxido nítrico inalatório e as novas modalidades ventilatórias, mudou o curso das doenças pulmonares com o surgimento de novos padrões de comprometimento dos pulmões, assim como se observou uma mudança nas características da população atendida nas unidades neonatais, havendo predomínio de crianças cada vez mais imaturas, o que alterou, consequentemente, a assistência fisioterapêutica ${ }^{(2,3)}$.

Observou-se a não-utilização de medicamentos como sedativos e/ou analgésicos na população estudada, já que essa não é a rotina clínica da unidade neonatal. O uso de anticonvulsivantes só é indicado quando se observam quadros

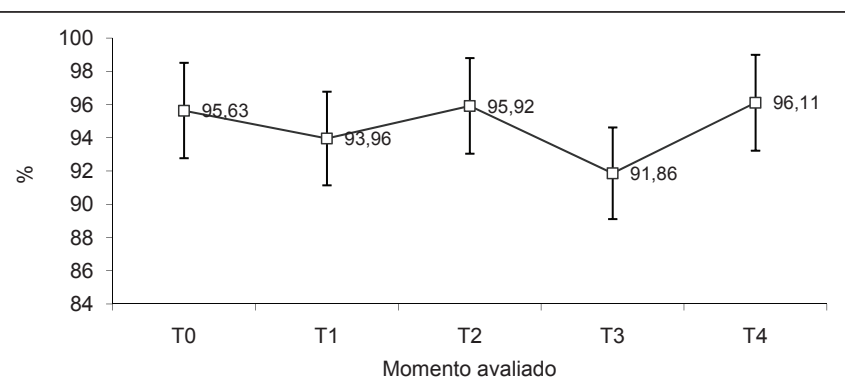

ANOVA MR: T2xT3 $p<0,001 ;$ T3xT4 $p<0,001$.

Figura 3 - Média da saturação de oxigênio dos recém-nascidos estudados, de acordo com o momento avaliado.

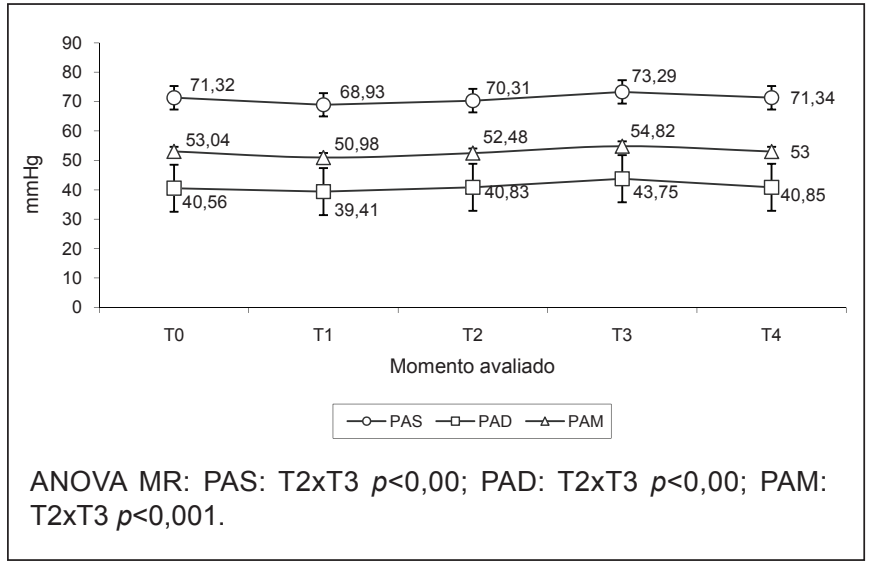

Figura 4 - Média da pressão arterial sistólica (PAS), diastólica (PAD) e média (PAM) dos recém-nascidos estudados, de acordo com o momento avaliado.

convulsivos, fato infrequente nesta população. A introdução de estimuladores do centro respiratório, como as xantinas (cafeína, aminofilina), se faz com a progressão do desmame da ventilação mecânica, geralmente com a adoção de parâmetros ventilatórios baixos (frequência respiratória menor que 20 rpm), o que não se observou nesta população pelo fato de o estudo ter se limitado à primeira semana de vida do $\mathrm{RN}$.

É bem conhecido o fato de que a hemorragia intracraniana peri-intraventricular ocorre com maior frequência nas primeiras 72 horas de vida nos RNPT com peso de nascimento inferior a $1500 \mathrm{~g}$. Inúmeros fatores intra e extravasculares podem contribuir para promover o sangramento ou o seu aumento, dentre eles, a persistência do canal arterial, a ventilação mecânica e, possivelmente, certos procedimentos como a aspiração traqueal e a própria fisioterapia respiratória ${ }^{(3,4)}$. Com essa preocupação em mente, seguiu-se a rotina do Serviço de Fisioterapia do Instituto da Criança (HC/FMUSP), que determina o início do tratamento fisioterapêutico somente após as primeiras 72 horas de vida nos 
RNPT de muito baixo peso, justificando-se, assim, o período estipulado para o início da coleta de dados. Foi protocolado o sétimo dia de vida para o término da coleta, compreendendo, desta forma, a primeira semana de vida e, portanto, o período mais crítico da evolução clínica desses RN.

No presente estudo, foram selecionados como parâmetros os valores da frequência cardíaca e respiratória, saturação de oxigênio e pressão arterial sistêmica, por serem representativos da função cardiopulmonar e, também, por serem utilizados rotineiramente nas unidades neonatais devido à facilidade de mensuração e baixo risco para o $\mathrm{RN}$ por serem medidas não-invasivas.

Em relação à frequência cardíaca (FC), observou-se que esta foi diretamente afetada pelos procedimentos fisioterapêuticos e de aspiração nos $\mathrm{RN}$ de muito baixo peso. Dados semelhantes foram encontrados nos estudos de Kelly et a ${ }^{(12)}$ e Main et $a l^{(13)}$, nos quais se observou aumento da frequência cardíaca após as manobras de fisioterapia e aspiração endotraqueal. A FC pode aumentar como mecanismo compensatório para manter o débito cardíaco ou pode diminuir em resposta à hipoxemia ${ }^{(14)}$.

Os achados deste estudo em relação ao ritmo cardíaco mostraram grande variabilidade nos $\mathrm{RN}$ estudados. Este fato pode ser explicado pela influência do desenvolvimento dos barorreceptores cardíacos, os quais são pobremente desenvolvidos nos RNPT extremos ${ }^{(15)}$. O barorreflexo se torna mais funcional com o desenvolvimento e maturação pós-natal, assim como a variabilidade da FC e a diminuição dos batimentos cardíacos são mais consistentes com a maturação da atividade autonômica cardiorreguladora que se processa a partir de 31 semanas de idade gestacional ${ }^{(15-17)}$.

O RN apresenta diferenças importantes em relação ao controle da respiração e frequência respiratória em comparação ao adulto. O RN é mais sensível aos estímulos periféricos, não possui uma boa coordenação entre seus músculos respiratórios e os músculos que controlam a permeabilidade das vias aéreas superiores e exibe um padrão de sono ainda imaturo, com predominância de sono REM. Além disso, mostra uma resposta aguda à hipóxia diferente, com diminuição importante do metabolismo, ventilação e temperatura corporal, o que lhe confere uma capacidade muito maior de sobreviver à agressão hipóxica ${ }^{(18,19)}$. Este fato pode explicar os resultados encontrados neste estudo: a frequência respiratória não apresentou grande variabilidade durante a intervenção de fisioterapia e a aspiração endotraqueal.

Um método simples, não-invasivo e rotineiramente disponível para avaliar a oxigenação do $\mathrm{RN}$ por avaliações periódicas e monitorização contínua é a saturação de oxigênio $\left(\mathrm{SatO}_{2}\right)$ através da oximetria de pulso. Os resultados deste estudo demonstraram uma diminuição maior da saturação de oxigênio após a aspiração endotraqueal. Resultados semelhantes foram relatados por Kelly et al ${ }^{(12)}$ e Main $e t ~ a l^{(13)}$, que mostraram redução estatisticamente significativa dos valores da $\mathrm{SatO}_{2}$ após o procedimento de aspiração da cânula endotraqueal. Tal situação poderia ser justificada pelo fato de que, nos estudos citados, foi utilizado o sistema aberto de aspiração, no qual o paciente é desconectado da ventilação mecânica para o procedimento; desta forma, o aporte pressórico e de oxigênio para o paciente é interrompido. Uma maneira de realizar o procedimento de aspiração sem a desconexão do paciente do ventilador mecânica seria através do sistema fechado de aspiração. Contudo, estudos recentes compararam o sistema aberto com o sistema fechado de aspiração e não encontraram diferenças entre os dois, além de o custo do sistema fechado ser mais elevado ${ }^{(20-22)}$

A determinação da pressão arterial deve ser realizada frequentemente nos prematuros sob cuidados intensivos, já que as oscilações na pressão arterial sistêmica são diretamente proporcionais às oscilações do fluxo sanguíneo cerebral nos prematuros devido à falha do mecanismo de autorregulação e estão associadas ao desenvolvimento da hemorragia periintraventricular ${ }^{(23-25)}$. Os resultados deste estudo mostraram que os procedimentos fisioterapêuticos empregados não podem ser considerados prejudiciais para os RNPT; porém, a aspiração endotraqueal teve maior repercussão sobre a pressão arterial sistêmica. Gronlund $e t a l^{(25)}$, Mosca $e t$ al ${ }^{(26)}$ e Tsuji et a ${ }^{(27)}$ demonstraram como as variações da pressão arterial estavam associadas às variações da oxigenação intravascular cerebral em prematuros criticamente doentes.

Apesar de as variáveis estudadas terem apresentado significância estatística em alguns dos momentos avaliados, todos os valores permaneceram em intervalos considerados fisiológicos. Contudo, a não-equivalência da significância estatística e da importância clínica destes resultados deve ser considerada criteriosamente; um resultado significativo estatisticamente muitas vezes pode não ser considerado clinicamente importante, assim como é verdadeiro o inverso ${ }^{(28)}$. Desta forma, torna-se imprescindível que o papel do fisioterapeuta dentro das unidades neonatais seja reformulado, deixando de ser somente o profissional que aplica técnicas para a manutenção da higiene brônquica e passe a ser também o profissional que pesquise e questione o real valor da fisioterapia respiratória, procurando respostas para questões como: em que situações clínicas os recursos de fisioterapia podem ser deletérios? É possível identificar situações clínicas que limitem o uso dos recursos fisioterapêuticos? 
Assim, neste estudo a respeito da influência da fisioterapia respiratória sobre a função cardiopulmonar em $\mathrm{RN}$ pré-termo de muito baixo peso submetidos à ventilação mecânica durante a primeira semana de vida, tanto os procedimentos de fisioterapia respiratória quanto o procedimento de aspiração endotraqueal não influenciaram de forma significativa a função cardiopulmonar, sugerindo que, quando bem indicados e realizados, não comprometem a estabilidade clínica dos RN pré-termo.

\section{Referências bibliográficas}

1. Nicolau CM. Estudo das repercussões da fisioterapia respiratória sobre a função cardiopulmonar em recém-nascidos pré-termo de muito baixo peso. [tese de mestrado]. São Paulo (SP): FMUSP; 2006.

2. Hudson RM, Box RC. Neonatal respiratory therapy in the new millennium: does clinical practice reflect scientific evidence? Aust J Physiother 2003;49: 269-72.

3. Juliani RCTP, Lahoz ALC, Nicolau CM, Paula LCS, Cunha MT. Fisioterapia nas unidades de terapia intensiva pediátrica e neonatal. Programa Nacional de educação continuada em Pediatria- PRONAP-SBP 2003/2004;30:1014-6.

4. Silveira RC, Procianoy RS. Ischemic brain damage in very low birth weight preterm newborn infants. J Pediatr (Rio J) 2005;81(Suppl 1):S23-32.

5. Flenady VJ, Gray PH. Chest physiotherapy for preventing morbidity in babies being extubated from mechanical ventilation. Cochrane Database Syst Rev 2002:CD000283.

6. Halliday HL. What interventions facilitate weaning from the ventilator? A review of the evidence from systematic reviews. Paediatr Respir Rev 2004;5 Suppl A:S347-52.

7. Bagley CE, Gray PH, Tudehope DI, Flenady V, Shearman AD, Lamont A. Routine neonatal postextubation chest physiotherapy: a randomized controlled trial. J Paediatr Child Health 2005;41:592-7.

8. Lewis JA, Lacey JL, Herderson-Smart DJ. A review of chest physiotherapy in neonatal intensive care units in Australia. J Pediatr Child Health 1992;28: 297-300.

9. Al-Alaiyan S, Dyer D, Khan B. Chest physiotherapy and post-extubation atelectasis in infants. Pediatr Pulmonol 1996;21:227-30.

10. Bloomfield FH, Teele RL, Voss M, Knight DB, Harding JE. The role of neonatal chest physiotherapy in preventing post extubation atelectasis. J Pediatr 1998;133:269-71.

11. Santos AM. Monitorização do recém-nascido com doença pulmonar. In: Alves Filho N, Trindade O, editores. Clínica de perinatologia: aparelho respiratório em neonatologia - Parte I. Rio de Janeiro: Medsi; 2001. p. 17-30.

12. Kelly MK, Palisano RJ, Wolfson MR. Effects of a developmental physical therapy program on oxygen saturation and heart rate in preterm infants. Phys Ther 1989;69:467-74.

13. Main E, Castle R, Newham D, Stocks J. Respiratory physiotherapy vs. suction: the effects on respiratory function in ventilated infants and children. Intensive Care Med 2004;30:1144-51.

14. Hanna BD, Nelson MN, White-Traut RC, Silvestri JM, Vasan U, Rey PM et al. Heart rate variability in preterm brain-injured and very-low-birth-weight infants. Biol Neonate 2000;77:147-55.

15. Mazursky JE, Birkett CL, Bedell KA, Ben-Haim AS, Segar JL. Development of baroreflex influences on heart rate variability in preterm infants. Early Hum Dev 1998;53:37-52.

16. Sahni R, Schulze KF, Kashyap S, Ohira-Kist K, Fifer WP, Myers MM Maturational changes in heart rate and heart rate variability in low birth weight infants. Dev Psychobiol 2000;37:73-81.

17. Lopes JM, Almeida VL, Sant'anna GM. Controle da respiração no recémnascido. In: Alves Filho N, Trindade O, editores. Clínica de perinatologia: aparelho respiratório em neonatologia - Parte I. Rio de Janeiro: Medsi; 2001. p. 1-15.

18. Holditch-Davis D, Scher M, Schwartz T. Respiratory development in preterm infants. J Perinatol 2004;24:631-9.

19. Cordero L, Sananes M, Ayers LW. Comparison of a closed (Trach Care MAC) with an open endotracheal suction system in small premature infants. J Perinatol 2000;20:151-6.

20. Rieger H, Kuhle S, Ipsiroglu OS, Heinzl H, Popow CN. Effects of open vs. closed system endotracheal suctioning on cerebral blood flow velocities in mechanically ventilated extremely low birth weight infants. J Perinat Med 2005;33:435-41

21. Copnell B, Tingay DG, Kiraly NJ, Sourial M, Gordon MJ, Mills JF et al. A comparison of the effectiveness of open and closed endotracheal suction. Intensive Care Med 2007;33:1655-62.

22. Kuban KC. Hemorragia intracraniana. In: Cloherty JP, Stark AR, editores. Manual de neonatologia. 4. ed. Rio de Janeiro: Medsi; 2000. p. 533-43.

23. Volpe JJ. Intracranial hemmorrhage: periventricular intraventricular hemmorrhage of the premature infant. In: Volpe JJ. Neurology of the newborn. $3^{\text {rd }}$ ed. Philadelphia: Saunders; 1995. p. 403-63.

24. Harding JE, Miles FK, Becroft DM, Allen BC, Knight DB. Chest physiotherapy may be associated with brain damage in extremely premature infants. J Pediatr 1998;132:440-4.

25. Grönlund JU, Korvenranta H, Kero P, Jalonen J, Välimäki IA. Elevated arterial blood pressure is associated with peri-intraventricular haemorrhage. Eur $\mathrm{J}$ Pediatr 1994;153:836-41.

26. Mosca FA, Colnaghi M, Lattanzio M, Bray M, Pugliese S, Fumagalli M. Closed versus open endotracheal suctioning in preterm infants: effects on cerebral oxygenation and blood volume. Biol Neonate 1997;72:9-14.

27. Tsuji M, Saul JP, du Plessis A, Eichenwald E, Sobh J, Crocker R et al. Cerebral intravascular oxygenation correlates with mean arterial pressure in critically ill premature infants. Pediatrics 2000;106:625-32.

28. Sola A, Dieppa FD, Rogido MR. An evident view of evidence-based practice in perinatal medicine: absence of evidence is not evidence of absence. J Pediatr (Rio J) 2007;83:395-414. 